\title{
Frontières
}

\section{Cosmétique des humeurs}

\section{L'antidépresseur dans l'espace social}

\section{Christian Saint-Germain}

Volume 16, numéro 1, automne 2003

Remède ou poison?

URI : https://id.erudit.org/iderudit/1073766ar

DOI : https://doi.org/10.7202/1073766ar

Aller au sommaire du numéro

Éditeur(s)

Université du Québec à Montréal

ISSN

1180-3479 (imprimé)

1916-0976 (numérique)

Découvrir la revue

Citer ce document

Saint-Germain, C. (2003). Cosmétique des humeurs : l'antidépresseur dans

l'espace social. Frontières, 16(1), 85-90. https://doi.org/10.7202/1073766ar

Ce document est protégé par la loi sur le droit d'auteur. L'utilisation des services d'Érudit (y compris la reproduction) est assujettie à sa politique d'utilisation que vous pouvez consulter en ligne.

https://apropos.erudit.org/fr/usagers/politique-dutilisation/
Cet article est diffusé et préservé par Érudit.

Érudit est un consortium interuniversitaire sans but lucratif composé de l'Université de Montréal, l'Université Laval et l'Université du Québec à Montréal. Il a pour mission la promotion et la valorisation de la recherche. https://www.erudit.org/fr/ 


\section{COSMÉTIQUE DES HUMEURS \\ L'antidépresseur dans l'espace social}

\section{L'HISTOIRE HUMAINE N'EST QUE L'HISTOIRE DE L'ASSERVISSEMENT QUI FAIT DES HOMMES, AUSSI BIEN OPPRESSEURS QU'OPPRIMÉS, LE SIMPLE JOUET DES INSTRUMENTS DE DOMINATION QU'ILS ONT FABRIQUÉS EUX-MÊMES, ET RAVALE AINSI L'HUMANITÉ VIVANTE À ÊTRE LA CHOSE DE CHOSES INERTES}

WEIL, 1995, P. 61

\section{ARE YOU READY FOR PROZAC WEEKLY ?1}

\section{Christian Saint-Germain, Ph.D., professeur titulaire, Département de philosophie, UQÀM.}

\section{L'ÉTAT ANXIEUX}

Le dernier 3 du lettrage lumineux de 3 heures 33 apparaît sur le cadran électronique d'une chambre sombre: Un homme se lève brusquement à 90 degrés dans son lit, comme si par de vives secousses il venait d'échapper à l'inflexible main d'un tortionnaire et avait pu, pour un moment, remonter sa tête plongée dans un récipient d'eau glacée.

Nuque mouillée de sueur, respiration d'une ampleur démesurée - réminiscence de l'irruption fotale dans l'oxygène brûlant. L'homme qui s'éveille commence à comprendre la capacité sérotoninergique de l'antidépresseur à produire des rêves d'une rare consistance, expérimentés dans le direct du sommeil par l'utilisateur. Il était en rêve au milieu d'un musée fermé qui n'avait gardé en veilleuse que l'éclairage requis pour alimenter d'une lueur chacun des tableaux de ses galeries. Se levant dans l'atmosphère contrôlée de ces intérieurs douillets, il s'étonna d'apercevoir sur les murs des scènes de sa vie ayant l'apparence lointaine d'avoir été conçues par de petits maîtres anciens, des miniatures hollandaises représentant simplement des moments de son enfance, jusqu'à sa vie d'adulte consacrée par l'explosion d'huile et de pastels d'un Francis Bacon. Comme à l'occasion d'un rêve freudien, deux conservateurs se tenaient à l'entrée d'une salle et n'étaient point troublés par ce visiteur nocturne. Il crut y reconnaître ses deux parents morts depuis longtemps mais qui étaient, à la faveur du stupéfiant effet de ces molécules, sur le seuil d'une salle pendant toute la durée de sa visite.

Non moins surprenante avait été sa rencontre avec un appétit qui l'arrachait de son sommeil matinal à la recherche de nourritures sucrées, de pains. Ses yeux cernés l'auraient rendu confondable avec un raton laveur urbain. L'apparition temporaire d'une sexualité chérubinique, le débarras de toute pensée à caractère sexuel coïncidaient avec la manière dont il appuyait sa tête sur le duvet de pêche d'un petit ventre féminin.

Il lui semblait vivre prisonnier d'une maison où il ne serait plus possible d'interrompre le signal d'une alarme stridente. Devenu le cambrioleur familier de sa propre demeure vide et s'y habituant. Soumis à l'irrépressible désir de faire quelque chose, d'acheter, d'aller voir, de changer de poste, de vérifier, de ne plus tenir en place. Finalement expérimenter les premiers moments d'intimité entre une dilution de peroxyde d'hydrogène et la lèvre boursouflée d'une plaie béante.

\section{L'ANTICOAGULANT \\ DES IDÉES NOIRES}

Un grand nombre de personnes en Amérique du Nord s'éveillent au petit matin l'esprit barbouillé de réminiscences, de colères. Ce désarroi intime plutôt que de se dissiper dans l'aube crépitante - des réveils digitaux, des premières déceptions des enfants, des travaux en retard laissés derrière soi la veille - les poursuit tout le jour durant : en déjeunant, au moment de quitter du regard les indices de la Bourse et les prévisions de la météo pour s'installer devant l'horizon bleu d'autres écrans.

Des heures durant, chacun subit le tutoiement des écrans dans un univers qui renvoie en miroir l'exigence d'une réponse immédiate. Il ne s'agit plus tant du déplacement d'une main-d'œuvre mais de déplacer des corps de surveillance, des grappes d'yeux, des regards disciplinés par la logique des tubes. Lultra-domestication de l'esprit par l'œil, cette dextérité à comprendre où il faut placer le curseur et entrer les codes, commande en soi l'exigence simultanée de deux aptitudes diamétralement opposées : la suppression de tous les mouvements inutiles du corps et, dans un même moment, une hyperactivité de l'esprit acceptant de répondre sans relâche aux questions surgissant des signes affichés en surimpression sur les moniteurs. Les premiers moments de cet apprentissage, de ce dressage de l'œil, donnent l'impression au corps qu'il subit de brusques freinages, frappant un mur de pixels, qu'il est brutalement empêché par la commission d'une action impertinente à l'écran. Le «non» brutal des machines intelligentes surgit comme le coup de laisse donné sur l'étrangleur d'un chien dissipé. 
Le filet du monde se resserre dans le grésillement d'informations à traiter comme le jaune d'œuf se contracte sur la plaque brûlante d'une chaîne de restauration rapide. Il suffit ensuite d'observer les embouteillages dans le lacis des centres-villes comme un infarctus massif d'impatience, d'irritation, de musiques et d'informations de toutes natures. Accroissement des rythmes, des tempi dans un tonus qui n'a de consistance que pour lui-même et emporte l'être occupé par l'étrange jouissance de ce prurit de l'esprit. Suivre un crescendo de contrariétés, de retards, d'énervements produits par tous les objets qui nous tiennent désormais à leur merci.

La mise en forme de l'ordinaire atteint une densité hiéroglyphique. La précipitation vers des échéances, la prolifération des agendas électroniques commandent des sujets qui y naviguent une qualité particulière d'attention, de mémoire brève, procédurale, précisément requise par cette nuée d'injonctions fonctionnelles. Atlas ne soutient plus le monde depuis la puissance de ses bras et de ses jambes mais plutôt par de formidables coups de tête comme un joueur de foot redirige vers ses coéquipiers le ballon au jeu. Des dizaines de tâches, le to do list des machines accompagné de l'archi-texte publicitaire surmoïque, le filigrane convulsif de l'inconscient collectif : Just do $i t^{2}$, The best suprise is no suprise ${ }^{3}$. Cette ahurissante sollicitation de l'œil et de l'esprit laisse sans repos et ne s'arrêtera pas de sitôt. L'être humain transite désormais dans un univers de minuscules icônes, un jardin zen d'indications, de données de toutes espèces jusqu'à la consomption du soi comme habitacle narcissique contenant ce pèlerinage. Nous habitons le boxing day du désespoir. C'est le "destin de l'excitation» dans l'être pétrifié qui réclame du dispositif qui le produit la délivrance d'exquises potions, anticipe le redressement humoral par métabolisation, magie non métaphorique. L'inapaisé, la transe invisible, le sans parole d'une jouissance qui mène à rouler à toute allure au sortir du bureau, à revérifier le canon d'une arme de chasse, à espérer d'une scène pornographique qu'elle débarrasse du surplus de tension incommunicable ${ }^{4}$. Éprouver le fait divers d'une existence à soi dans une société de consommation, dans le pur présent sans présence.

Discipline de la vision, la géographie intérieure des machines, de leur dépliement logique pour l'action finit par être parfaitement intériorisée par l'utilisateur mieux qu'il ne se comprend lui-même. L'ordonnancement hiérarchique des procédures qui conduisent obligatoirement à l'accomplissement d'une tâche s'inscrit dans un réseau de finalité prévisible. Mécanisme rassurant des langages sans ombre, des engrenages qui réalisent un itinéraire de conséquences formelles. Cette modélisation est rapidement importée par l'utilisateur comme le décalque de son propre esprit tributaire à son tour de cette exacte malléabilité, découpée en zones d'interventions imaginaires ou réelles.

Indivision, connexité des prothèses du soi qui ne remplissent plus seulement des fonctions mais extirpent de toute condition naturelle jusqu'à la supplantation de l'horizon initial. Le dépôt de l'identité de soi ne compte que pour le plaisir de sa dilapidation. Autrement dit, qui a connu le clavier d'un ordinateur ne peut voir dans celui qu'offrait un dactylo qu'un instrument de torture médiéval, le supplice exercé par une rotule métallique et son empreinte de suie sur le corps d'un cylindre.

Non seulement il n'est plus possible de revenir en arrière après avoir expérimenté ce que les technologies induisent, mais celles-ci introduisent à une telle dimension projective qu'elles inclinent à n'avoir jamais voulu se connaître autrement que comme ce vers quoi elles nous propulsent. Futuration de l'identité. Une sorte de fission de chacun des atomes qui composent l'ici et le maintenant, le hic et nunc. La modernité s'inscrit en porte-à-faux à l'égard d'une " primitive santé » des sujets. Maladies mentales et inconforts psychiques ne seraientils que des atavismes physiologiques, des retards du corps quant aux problèmes inédits qui, de toutes parts, se disputent l'esprit? Déphasage des vieux systèmes limbiques et de leur architecture Fight or Flight. L'antidépresseur vise à reconfigurer le comportement humoral de l'hypothalamus au même titre que le technicien informatique dans le système Mac cherche à relancer une machine qui plante en affichant un Sad Mac ou dans le système X exhibant un Kernel Panic. Il ne s'agit plus de suivre le sens du symptôme mais de convenir que ce qui produit les comportements acceptables ou intelligents n'a rien de commun avec la représentation philosophique de l'intelligence ou de la volonté.

\section{LA COMPOSITION MOLÉCULAIRE DES RÉVOLUTIONS}

Plus encore que les idéologies, le médicament constitue le catalyseur par excellence des métamorphoses radicales de l'espace social. Un accélérateur de mutations irréversibles. Il n'est qu'à considérer les effets des anovulants sur les prédications et les morales des organismes constitués pour produire tous ces bavardages savants avec moult apparats et costumes d'un autre âge. Le fatras ostentatoire et les mécaniques révérencieuses se dissipent comme le dernier salut d'une troupe de théâtre constituée par de vieux comédiens invertis.
Trente années de fidélisation à des concentrés œstrogéniques ont mis un terme à des siècles de captivité des femmes à la fonction reproductrice et à leur assignation à un ordre social dont la staticité paraissait immuable. Plus encore, l'irruption de cette technologie pharmaceutique nous aura obligés à la réorganisation des rapports entre les sexes et mis en cause la place, le nombre et finalement l'intérêt même de faire des enfants. Or, aucune révolution de papier, d'idées ou de revendications émancipatoires n'y serait parvenue sans cet adjuvant moléculaire.

Les technologies portent bien davantage à conséquence sur la vie sociale que ne sauraient le faire les consensus entre les hommes, d'hypothétiques évolutions des mentalités ou de poignantes délibérations. La portée des molécules sur les comportements exproprie d'un coup les attachements aux formes arrêtées, les conceptions du bonheur des époques antérieures. Comme en informatique où un système d'exploitation "écrase» le précédent. Les êtres humains restent tout de même attachés pour leur plus grand malheur aux images anciennes. À l'idée de famille, de procréation qu'ils tentent de perpétuer dans les décombres des anciennes configurations sociales. Ils habitent à leurs dépens dans la détresse de cette fracture anomique. Squatter du provisoire, de l'incertain. Il n'est pas de coïncidence immédiate entre les représentations individuelles du bonheur, de la sécurité et l'intraitable état de fait imposé par le poids du comprimé dans la misérable balance des choix individuels.

Le désir d'enfant, par exemple, paraît dans une société de consommation avancée la conséquence d'un archaïsme pathétique ou l'expression d'un luxe inouï. Un peu à la manière des banlieusards qui aspergent des heures durant par une belle journée ensoleillée, une grosse cylindrée avec de l'eau potable.

Le médicament redresse tout autant les humeurs des sujets que leurs érections défaillantes. Il importe d'entrevoir la montée d'une communauté sous influence, non plus seulement soumise à un paysage médiatique publicitaire ou divertissant mais fournissant, d'abord sous prétexte médical, une gamme de produits permettant l'élusion du soi narratif par de petites prothèses coalescentes. Nul intérêt de guérison; seulement sentir que ce qui confortait l'impression chronologique d'urgence s'est brusquement interrompu sous l'effet du comprimé. Le sentiment de délivrance, l'apesanteur dans d'immenses globules d'oxygène et la remontée dans toute la lumière après une nuit de 12 heures. Mais aussi, pour l'avenir, la suggestion d'un agenda électronique qui, eu égard au classement des tâches journalières, 
proposerait des doses et des molécules utiles pour produire selon les circonstances : l'attention diffuse, l'affabilité, l'enthousiasme festif, la concentration maximale comme modulation de l'arrière-fond du soi.

\section{L'INFLUENCE \\ CHIMIQUEMENT PURE}

De tous les objets produits par la société de consommation, c'est le médicament, en particulier l'antidépresseur, qui concentre autour de lui, la plus importante offensive biopolitique intégrée aux nouvelles stratégies de contrôle des conduites individuelles. À la fois généré par de puissantes industries pharmaceutiques, inscrit tout à la fois dans une généalogie moléculaire et dans un imposant dispositif de recherche, l'antidépresseur exerce une influence imaginaire grâce surtout à la confiance thérapeutique de voir une partie récalcitrante de soi être mise au pas sans médiation narrative.

Un déplacement du champ des imaginaires vers le dépôt d'autorité désormais incarné par la médecine moderne remplace la chaîne sacramentelle des artefacts de la foi. Un important capital flottant de crédulité a été récupéré par la fonction normative du discours médical. Dans cette optique, santé et salut produisent des attentes qui ne peuvent s'actualiser pleinement qu'à l'occasion de l'incorporation d'un objet partiel : saintes espèces ou par prescription de molécules révolutionnaires.

Dans l'univers pharmaceutique, le comprimé antidépresseur n'est pas un médicament parmi d'autres, pas plus que l'hostie dans l'imaginaire catholique n'est un résidu céréalier d'une forme singulière. À vrai dire, le «placebo » dont les effets sont tant redoutés par l'industrie est lui-même issu de la tradition liturgique (dire un placebo). Intersection d'influences diverses, Philippe Pignarre note que : «Influence du médecin sur le patient, influence du commanditaire de l'étude sur le médecin expérimentateur: on ne sait jamais, dans une telle situation, qui contraint l'autre et comment ce type de contraintes pourrait être démêlé au profit de la "vraie réalité" » (Pignarre, 1997, p. 29).

L'objet s'enrichit donc à chacune des étapes de sa diffusion d'un pli de confiance : origami moléculaire. Il importe d'esquisser l'histoire du comprimé. Histoire de sa forme oblongue, de la capsule, de la gélule, des entités sublinguales telles de minuscules têtes d'épingles. Le choix judicieux des «bleus piscine» pour l'Immovane ${ }^{\circledR}{ }^{5}$, les couleurs du Paxil ${ }^{\circledR}$ variant selon les dosages ou encore l'Effexor $\AA$ à l'image d'une bombonne rose, une bombe de tranquillité des années 1960 après une sortie dans le Viêtnam intérieur. Extase des gélatines et des adjuvants : petites montres molles pour contrer les morsures du temps extérieur qui dévore invinciblement le tour des yeux et le dessus des mains.

Cette délicate esthétique du comprimé ne manque pas de charme et n'est pas ellemême étrangère aux effets sur les microclimats de la subjectivité. Elle constitue l'esthétique du quotidien, concentre le salut en milligrammes et en doses, procure l'intime délivrance d'être soi sans pour autant devenir autre. D'être au milieu des milles éclats de verre du présent : dernier maillon d'une chaîne de catastrophes.

Il en va des assises d'une pyramide de confiance, pierre angulaire sur laquelle reposent des millions de personnalités nord-américaines qui confient leurs expectatives de bonheur à la quotidienneté des technologies. La prise matinale du comprimé marque l'appartenance à une communauté de fidèles anonymes de la révélation statistique du DSM-III ou IV $^{6}$ à travers l'Amérique. Une sorte de « marteau

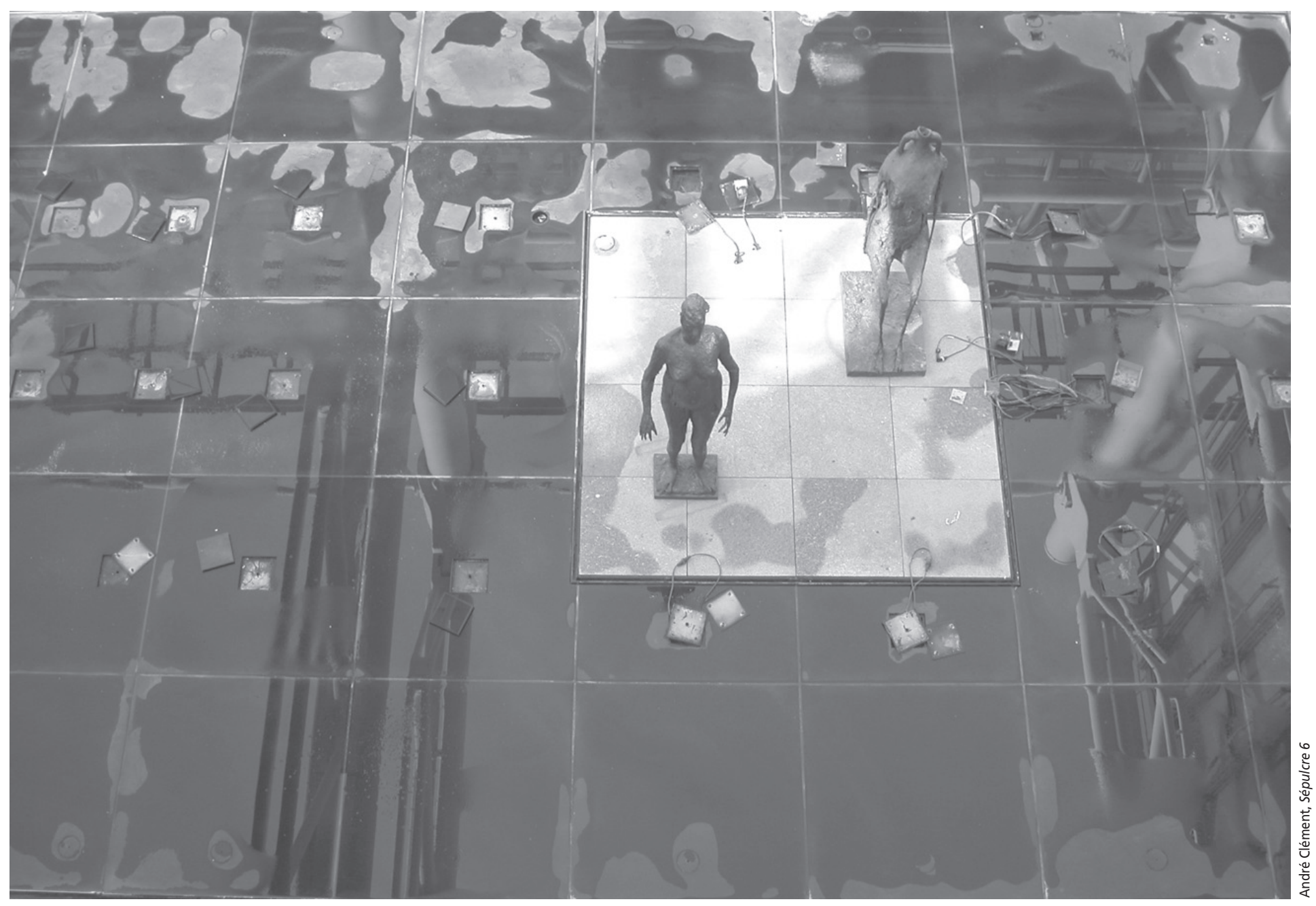


des sorcières » pour contrer la production de pensées atypiques, dissonantes ou envahissantes. L'analogie avec le fonctionnement religieux peut fort bien accommoder l'organigramme qui conduit à la production de ces luxueux projectiles pour se prémunir des infestations par les esprits chagrins, soulager les suppliciés de l'insomnie. Sommes-nous prêts pour le Prozac $\AA$ hebdomadaire, à libération lente, rappelle la publicité?

\section{ÉPITAPHE DE LA NARRATION}

Dans les revues médicales qui s'adressent aux psychiatres, la galerie des portraits le confirme sans détour: majoritairement, des femmes soucieuses y ont retrouvé le sourire et marchent désormais côte à côte avec mari et enfants, dans le rétablissement de la communication et de la confiance. L'antidépresseur fait saillir la collusion toujours plus grande entre la découverte $d u$ bonheur et sa réduction à l'apparente normalité statistique des comportements. Simplification des incertitudes philosophiques et des quêtes: sublime raccourci qui mène au travail fin sur le remaniement du paysage intérieur.

Une société qui a inventé le bonheur ne peut concevoir que des personnes souffrant de troubles envahissants de la personnalité ou du comportement ne puissent s'y sentir heureux ou ne point y trouver leur place. Il existe désormais un tel désintérêt pour l'introspection, une telle abondance de confessions. Le présent insiste dans chaque heure qui passe pour arracher le sujet à quelque travail de recollection comme la force $g$ amène les corps vers l'intérieur de cette cascade d'images, d'impressions par l'effet d'une trame de sollicitations tactiles, visuelles générée par l'environnement d'un consommateur.

C'est que cette exigence d'un soi performatif convoque tantôt l'expérience dépressive ou son revers, celle de l'anxiété généralisée. C'est à ces deux stations ou à ces échangeurs que la molécule pharmaceutique intervient pour rétablir, pour relever par-dessus ce marché d'impératifs lilliputiens, le sujet de l'affairement. Le temps comprimé, c'est le temps du comprimé. Zoloft (sertraline HCI) Because each day counts $^{7}$.

À cette enseigne, un pan entier d'explication des comportements, des malaises ne passe plus par la narration, le transport de la plainte. Le malaise psychique est retourné à l'adresse de son expéditeur (snail mail). La psychanalyse reste prisonnière de sa risible componction. Elle ne conserve de patients que la faune de ses admirateurs un peu comme les stations thermales et les villes d'eaux ont gardé pour un certain temps, malgré leur inanité curative, de fortunés touristes.
L'expression de l'intériorité des sujets ne peut plus échapper à cette disposition du monde du travail et de la santé puisque ce sont les institutions qui attestent pour les sujets eux-mêmes de la réalité de leur vécu et leur fournissent ensuite les mots, les explications plausibles. La compréhension de leur malaise ne peut avoir lieu que dans le cadre des moyens mis à leur disposition par le dispositif médical pour accéder à la reconnaissance. Comme on dit «traduire en justice » pour décrire la soumission physique et linguistique imposée à un prévenu par l'appareil judiciaire. Or, ce qui du point de vue du sujet souffrant paraît appartenir à sa nature intime ne trouvera jamais de sens ailleurs que par le modèle dans lequel le fait de "souffrir psychiquement» est absorbé par un contexte médical. « Les faits ne se constituent qu'en relation avec les critères qui permettent de les constater » (Habermas, 1976, p. 14). Dans cette optique, il n'existe pas de subjectivité souffrante mais des comportements et des vécus qui correspondent à une densité statistique repérable. Se dissipe l'illusion d'une histoire personnelle, d'un arrimage au caprice du signifiant.

Le modèle thérapeutique n'est jamais en rupture idéologique avec la société qu'il prétend servir en intervenant auprès de ceux de ses membres dont il escompte obtenir la guérison. Parfaitement soluble dans l'imaginaire économique et religieux selon les époques, il épouse la plasticité des expectatives et des anticipations de la délivrance par des sujets consentants. Il fonctionne d'autant mieux que ceux qui $\mathrm{y}$ participent n'ont plus le moindre soupçon quant à l'acuité scientifique de leur péroraison consensuelle. Il suffit d'admirer la représentation des échanges synaptiques utilisée le plus souvent dans la publicité des antidépresseurs pour convaincre leurs usagers que le travail de la molécule saura les purger de leur morosité. L'important n'est pas tant que les choses aient lieu selon le dessein des étreintes synaptiques (les baisers profonds mais brefs des terminaisons nerveuses) mais que patients et prescripteurs conviennent qu'à cet endroit spécifique de l'anatomie, le courant passe.

Au-delà des modélisations biochimiques, la plus délicieuse médiation réside dans le " packaging » des produits, le choix du nom, les couleurs suggestives (un somnifère ne sera jamais rouge pompier). Un peu à la manière des aliments préparés pour les chats domestiques, il importe que l'acheteur qui ne porte ni moustache ni fourrure puisse y reconnaître à l'ouverture de la conserve les effluves viandeuses, pour imaginer que son compagnon zébré y retrouve le fumet de quelque carnage minuscule. À cet égard, les compagnies pharmaceutiques constituent des «focus group» de psychiatres pour les conseiller dans le choix des teintes, des noms et le design de présentation. Une immense pastorale biochimique remplace les congrès eucharistiques des anciennes sociétés. Une rencontre annuelle des psychiatres américains recèle pour l'éthicien de précieuses indications quant au devenir des sociétés de consommation avancées, tout comme les rencontres de vétérinaires spécialisés dans le traitement des bovins laitiers ont toujours permis d'entrevoir les avancements des technologies de reproduction assistée ultérieurement adaptées à la fécondité des femmes.

\section{BONHEUR GRANULAIRE}

L'ordre médical dans son expression pharmaceutique apparaît le plus clairement dans sa dépendance à l'égard des représentations sociales de la normalité et du bonheur portées par l'idéologie productiviste dominante. Entre les inconfortables tricycliques (et les IMAO inhibiteurs de la monoamine oxydase) et les nouveaux recapteurs sélectifs de la sérotonine (ISRS) se redessinent, en l'espace des vingt dernières années, les formes nouvelles d'un rapport à soi et une redéfinition du fonctionnement de l'esprit. Dans une société de planification et d'ordre, les sujets sont tenus de s'administrer à eux-mêmes le correctif prescrit ou de prendre toutes les dispositions nécessaires à l'exercice de leurs fonctions et à la tenue de leur rôle. Le sujet devient pour lui-même un objet à soumettre. Sujet des contraintes d'autant moins visibles qu'elles sont, plus qu'à aucune autre époque, intériorisées, et désormais métabolisées. La communion est alors parfaite entre un ordre normatif infaillible et un sujet ne désirant que sa réhabilitation ou sa réintégration au sein de ce dispositif.

Matérialisation de l'idée même de promesse et de ses effets, l'antidépresseur tamise l'ensemble des sollicitations incongrues internes sur lesquelles le sujet n'a aucune prise. Cet artefact cristallise toute la force anticipative que recèle l'espoir d'une délivrance du soi souffrant. Il libère de l'étrangeté, délivre de l'exotisme du fonctionnement de l'esprit que rencontre fugitivement à l'occasion de malaises psychiques le patient présumé. Il interrompt les procédures accusatoires engagées par l'insistance de certaines pensées au détriment d'autres plus confortables et surtout moins invasives. Mieux encore il rétablit, en invoquant l'action sur des blocs neuronaux spécifiques, des échanges dont la monnaie des fluides (sérotonine) ne se retrouve pas en quantité suffisante sur des sites spécifiques du cerveau ou subit une taxation trop élevée par ses antagonistes. Il importe alors d'inhiber la recapture et d'empêcher la 
dégradation de la sérotonine (une monnaie forte dans le circuit économique du bonheur d'être soi) en y fixant un petit pécule dans la fente synaptique. Il s'agit d'instaurer une zone de libre-échange afin de poursuivre, sous le mode intime cette fois, l'utopie qui gouverne à l'extérieur du soi.

En matière de soins psychiques, "guérir » signifie normaliser par tous les moyens. Cautériser un surplus de rage, de détresse, de sentiment d'être au milieu de conditions aussi pérennes qu'absurdes qui traversent le sujet désarmé, résolu à n'en point connaître l'origine sociale. Les effets découverts des molécules sur les comportements dessinent rétrospectivement les exagérations pathologiques à traiter.

La confection de symptômes psychiques constitue la monnaie, la devise étrangère de cette conversion. Mieux encore, le patient se porte lui-même à la rencontre de cette bienveillance pharmacologique en y adaptant ses symptômes. Mikkel Borch-Jacobsen décrit cette singulière prévenance des patients en ces termes : «[...] la dépression moderne est, si l'on ose dire un effet secondaire des antidépresseurs. [...] En choisissant de

Plus encore, la recherche d'un éden pharmacologique s'inscrit dans le déficit croissant de la socialisation. Le refus de la médiation, du passage de la reconnaissance par le désir de l'autre homme, les institutions, commandent le raccourci du circuit de la récompense, l'autarcie apparente du toxicomane. Prévoir l'apparition du manque implique une suite d'opérations moins complexes que l'obtention toujours incertaine de l'assentiment d'autrui. Autogénération ad hoc du sujet moderne, désintérêt des processus de socialisation. Il s'agit d'imaginer une société où chacun regarde ailleurs, se détourne infiniment du regard d'autrui, où ce qui reste encore en commun est constitué de toutes les manières nouvelles de se voir sans jamais devoir se regarder. Apothéose des écrans et finalement remplacement du tissu social par des politiques de gestion assimilables à un Safe Injection Site $^{8}$, comme une ombre qui se précise sous nos yeux. Loin d'être une mesure d'exception, il s'agit du devenir inavouable des grandes agglomérations, de l'exemple même du rétrécissement des rapports de solidarité entre les êtres.

\section{DANS UNE SOCIÉTÉ DE PLANIFICATION ET D’ORDRE, LES SUJETS SONT}

TENUS DE S'ADMINISTRER À EUX-MÊMES LE CORRECTIF PRESCRIT OU DE PRENDRE TOUTES LES DISPOSITIONS NÉCESSAIRES À L'EXERCICE

\section{DE LEURS FONCTIONS ET À LA TENUE DE LEUR RÔLE.}

devenir dépressifs, ils semblent donc avoir activement calqué leurs symptômes sur les médicaments psychotropes réputés agir sur ces mêmes symptômes» (2002, p. 20).

Autrement dit, nous avons collectivement toutes les raisons du monde, tout intérêt à croire en l'existence de la rémission des malaises psychiques par métabolisation d'un agent chimique, comme naguère il allait de soi que la communion constituait une condition sine qua non du sujet croyant à son inclusion personnelle à la vérité du salut. Seul un comprimé peut encore nous sauver. Il n'est point de démenti scientifique, de falsifiabilité qui viendrait à bout de cette nécessité désormais consubstantielle de l'horizon des sociétés de consommation avancées. Le vernis scientifique qui dore la pilule du consentement au bonheur paraît bien mince en regard de son utilité absolue. L'être humain ne peut prétendre avoir modifié complètement son univers extérieur et espérer s'y retrouver sans lui-même s'y fondre avec l'assistance d'adjuvants nouveaux, sans aménager en contrepartie le « confort » d'un paysage intérieur. Terrassement égotique.

\section{TRAFICS D'INFLUENCE}

C'est précisément la finalité thérapeutique des substances psychoactives qui constitue le dernier rempart, ralentit l'acceptation comme corollaire de la vie en commun, la constitution d'états de conscience modifiés et planifiés. Cette dernière réticence place les sociétés dans la curieuse opposition entre les intérêts distincts de deux influentes industries: celle de la créativité pharmaceutique et, son opposé, l'industrie de la répression. Corps de police, associations syndiquées de chefs et de petits chefs, comédiens de l'ordre qui font leur pain et beurre de la chasse aux substances illicites. Toutes créatures surnuméraires de la fouille, des lucratives technologies de surveillance.

Bien que le législateur finisse par préférer taxer la jouissance (la pénalisation par amende plutôt que la criminalisation pour la possession simple de la marijuana dans la nouvelle législation canadienne) plutôt que de soutenir l'interdit, il garde l'intérêt de distinguer ontologiquement des substances. Or, le trouble à ces frontières tient au fait qu'il n'en va pas d'un partage autre que sémantique ou par effet de fiction juridique. L'Association médicale canadienne ne s'est pas trompée en refusant la prescription de la marijuana à des fins thérapeutiques. Elle a compris qu'il fallait maintenir cette distinction artificielle entre les substances. Elle a craint sans doute que le patient finisse par établir un parallèle entre le médecin prescripteur (commandité à distance par de puissantes organisations pharmaceutiques) et le petit revendeur de la rue, qu'il reconnaisse un " air de famille» entre des activités en apparence diamétralement opposées.

La prolifération des substances psychoactives s'inscrit dans un déficit de socialisation qu'aucune économie de marché n'arrive à corriger. Chacun des vécus séparés, chaque individu ne tient à l'ensemble que par l'épicerie à grande surface et le réseau d'aqueducs.

Il n'y a pas de différences ontologiques entre les diverses mixtures qui altèrent la perception de soi. Seulement peut-être une différence de degré, d'intensité. Le législateur aimerait découper des corridors normatifs étroits : s'abriter derrière de fallacieuses fictions quant au rapport à ces produits. Les antidépresseurs cessent progressivement de guérir des dépressions ou la définition de cette dernière s'est tellement élargie qu'elle cesse de signifier une pathologie spécifique. Ces médicaments sont désormais compris par les compagnies d'assurances comme des médicaments de confort. De la même manière qu'il n'est point nécessaire pour une femme de soutenir une activité sexuelle trépidante pour s'assurer de la prise quotidienne d'un anovulant. Une grossesse non désirée ou un vécu dépressif préventivement tenu à distance? L'absorption de substances psychoactives licites, dépendance supervisée, marque dans l'histoire de la domination des hommes, la mise en place d'un « hyperlien » qui ne renvoie pas à la consistance organique d'une pathologie avérée mais dispose bien davantage du malaise croissant de l'adaptation des sujets à la nature complexe de leur environnement. L'analyste Patrick Guyomard observe le rapport équivoque au substances psychoactives comme le chiffre même du mode d'appartenance des sujets à une société de consommation.

\footnotetext{
Dans une société où nous sommes tous des consommateurs, le toxicomane par-delà un statut ne devient-il pas le " super-consommateur » qui aurait trouvé le vrai-faux produit idéal, la solution finale et radicale au manque? [...] Il éviterait toutes les mésaventures et désillusions, toutes les contraintes et les dépendances qui obligent à passer
} 
par les autres comme les chemins plus ou moins sûrs et balisés que les sociétés offrent pour s'humaniser (Geberovitch, 2003, p. 10).

«6.43... le monde de l'homme heureux est un autre monde que celui du malheureux »(Wittgenstein, 1961, p. 172).

\section{LE PHARMACIEN-ROI}

Traditionnellement, la philosophie exerçait une juridiction sur les humeurs, il n'est qu'à penser aux visées descriptives et à toutes les conjonctures biliaires aristotélicienne sur la mélancolie. L'ensemble des prescriptions diététiques d'alors et les recherches d'équilibre par le travail sur soi ont le même effet sur la conscience moderne que l'ellébore de Molière avec lequel il fallait purger les commères, le perlimpinpin ou le millepertuis.

Peu souhaitent se retrouver devant leur vie comme une vieille femme démaquillée devant son miroir le matin ou le soir, ni non plus comme l'homme âgé inquiété par la pluie sur sa mauvaise teinture. L'antidépresseur est le fond de teint du temps, le tonus du désespoir dans une vie planifiée, rationnelle et sans surprise.

La plaquette calendrier du Paxil $\AA$ avec ses rainures solaires pâles annonçant son chlorydrate de paroxétine. Chaque jour 20 milligrammes d'une dose d'installation promet une paix, une indifférence nouvelle et adoucit les irritations hypothalamiques et amygdaliennes à l'intérieur des anciens cerveaux. Moins de terreur de vivre. À la manière des anovulants, un petit écrin contient le projet chronologique et paroxystique pour faire voir la vie sous un autre jour que celui affolé du patient. Il y est même question du cycle de trente jours pour lequel on demande au consommateur de faire part de ses impressions selon qu'il se trouve au début, au milieu ou à la fin de cette première frappe biochimique de ses centres cérébraux. Comment engourdir les sites d'un formidable syndrome prémenstruel commun aux dépressifs, à l'anxieux. Il en va d'un inconfort qui ne suit pas les conséquences d'une idée sombre, d'une conception philosophique mais qui place l'esprit dans le réel sous la palpitation ou l'écoulement d'un temps vivant, cruel, qui dévore les nerfs tout crus.

\section{Bibliographie}

BORCH-JACOBSEN, Mikkel (2002). Folie à plusieurs. De l'hystérie à la dépression, Paris, Les empêcheurs de penser en rond.

GEBEROVITCH, Fernando (2003). No satisfaction. Psychanalyse du toxicomane, Paris, Albin Michel.

HABERMAS, Jürgen (1976). Connaissance et intérêt, Paris, Gallimard.

PIGNARRE, Philippe (1997). Qu'est-cequ'un médicament? Un objet étrange, entre science, marché et société, Paris, Éditions de la Découverte.
SAINT-GERMAIN, Christian (2003). L'oil sans paupière. Écrire l'émotion pornographique, Sainte-Foy, Presses de l'Université du Québec, 104 p.

WEIL, Simone (1995). Réflexions sur les causes de la liberté et de l'oppression sociale, Paris, Gallimard.

WITTGENSTEIN, Ludwig (1961). Tractatus logico-philosophicus, Paris, Gallimard.

\section{Notes}

1. <http://www.prozac.com/>

2. Slogan de Nike.

3. Slogan de la chaîne hôtelière Holiday Inn.

4. Voir à ce propos notre récent ouvrage (Saint-Germain, 2003).

5. Plus exactement, comme le «Neptune pool» du Hearst Castle à San Francisco.

6. Manuel diagnostique et statistique des troubles mentaux.

7. <http://www.zoloft.com/> Your information resource for mood and anxiety disorders.

8. Safe Injection Site évoque davantage que ne peut le faire la désignation française de «piquerie» en ce que cette expression renvoie à l'idée même de sécurité, de surveillance et du contrôle carcéral des utilisateurs autant qu'à l'effacement progressif du concept d'illégalité. Le drogué devient un objet de préoccupation à partir du moment où il ne s'agit plus de marquer une attention prévenante ou une désapprobation morale mais de protéger le corps social contre la possibilité d'une contamination virale inséparable de l'usage répété de seringues souillées.

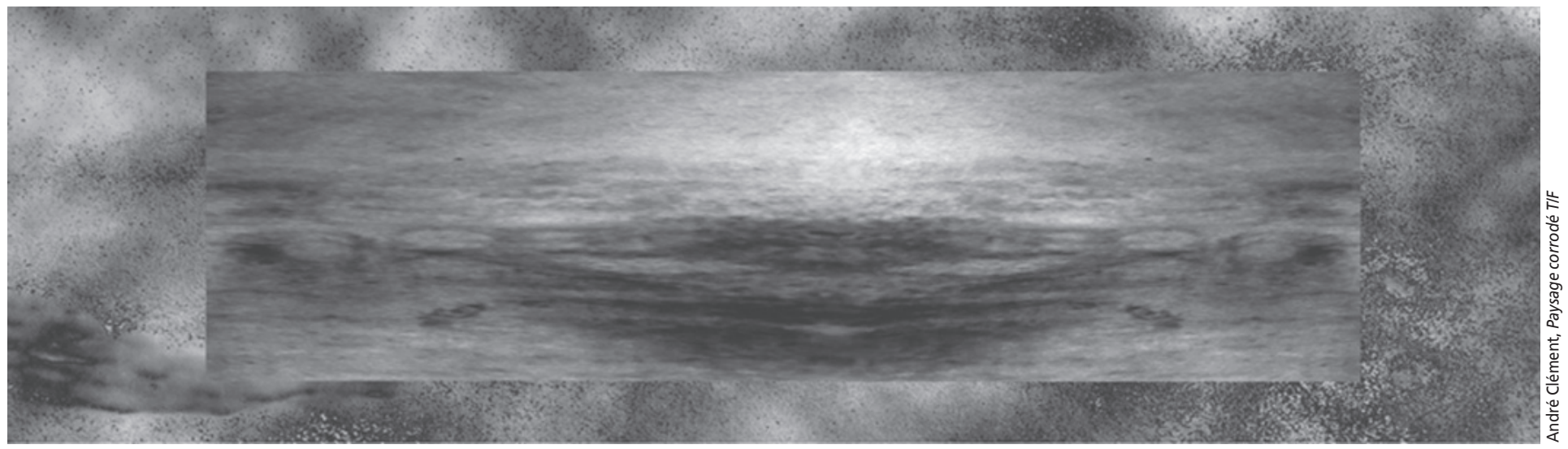

